\title{
Miniversal deformations of pairs of symmetric matrices under congruence
}

\author{
Andrii Dmytryshyn ${ }^{\mathrm{a}}$ \\ ${ }^{a}$ Department of Computing Science, Umeå University, SE-901 87 Umeå, Sweden.
}

Dedicated to Vladimir V. Sergeichuk on the occasion of his 70th birthday.

\begin{abstract}
For each pair of complex symmetric matrices $(A, B)$ we provide a normal form with a minimal number of independent parameters, to which all pairs of complex symmetric matrices $(\widetilde{A}, \widetilde{B})$, close to $(A, B)$ can be reduced by congruence transformation that smoothly depends on the entries of $\widetilde{A}$ and $\widetilde{B}$. Such a normal form is called a miniversal deformation of $(A, B)$ under congruence. A number of independent parameters in the miniversal deformation of a symmetric matrix pencil is equal to the codimension of the congruence orbit of this symmetric matrix pencil and is computed too. We also provide an upper bound on the distance from $(A, B)$ to its miniversal deformation.
\end{abstract}

Keywords: Symmetric matrix pair, Symmetric matrix pencil, Congruence canonical form, Perturbation, Versal deformation, Codimension

2000 MSC: 15A21, 15A63

\section{Introduction}

Finding a normal form to which all matrices $\widetilde{A}$, close to a given matrix $A$, can be reduced by certain transformation which smoothly depends on the entries of the matrix $\widetilde{A}$ is a challenging problem. In 1971 V.I. Arnold introduced such a normal form for matrices under similarity [2], see also [3, $\S 30 \mathrm{~B}]$, and called it a (mini)versal deformation. The prefix "mini-" is added if the number of independent parameters in the normal form is minimal. Now the notion of miniversal deformations has been extended to general [18, 22] and structured [7, 10, 11] matrix pencils, matrices of bilinear [10] and sesquilinear [11] forms, as well as to matrices under similarity over various fields [5, 21, 22].

Email address: andrii@cs.umu.se (Andrii Dmytryshyn) 
In this paper, we derive a miniversal deformation of a pair $(A, B)$ of symmetric matrices $\left(A=A^{T}\right.$ and $\left.B=B^{T}\right)$ under congruence; that is, a normal form with the minimal number of independent parameters, to which all pairs $(\tilde{A}, \tilde{B})$ of symmetric matrices close to $(A, B)$ can be reduced by congruence transformations smoothly depending on the entries of $\tilde{A}$ and $\tilde{B}$. Recall that a pair of $n \times n$ symmetric matrices $(A, B)$ is called congruent to $(C, D)$ if and only if there is a nonsingular matrix $S$ such that $S^{T} A S=C$ and $S^{T} B S=D$. The set of pairs of matrices congruent to a pair of symmetric matrices $(A, B)$ is called a congruence orbit of $(A, B)$. The codimension of the congruence orbit of a pair of symmetric matrices is equal to the number of independent parameters in the miniversal deformation of this pair and is computed in this paper too. We also bound the distance from the deformations to unperturbed pairs of matrices in terms of the norm of the perturbations. When talking about the previous results, we will sometimes use the term "matrix pencil" instead of "pair of matrices" (in the context of this paper these terms are equivalent).

Symmetric matrix pencils appear in a wide range of applications, including motion or vibration of structural systems [31, 33], viscous damping [17], network theory [4]. Often symmetric matrix pencils appear as a result of symmetric linearizations for symmetric matrix polynomials [1, 23]. Many of these applications require computing eigenstructures of matrix pencils, for example, via a structured staircase form for symmetric matrix pencils [6] as well as understanding the behaviour of these eigenstructures under low rank [30] and general perturbations, and that is where our miniversal deformations may be useful [7, 9, 20]. Moreover, based on the versal deformation theory, a constructive approach to determine the geometry of the singularities (orientation in space, magnitudes of angles, etc.) by constructing tangential cones to the stability domain is developed in [27, 29]. Some applications of miniversal deformations in control and stability theories can be found in [18, 26, 28]. In particular, miniversal deformations of symmetric matrix pencils can help us to construct their stratifications, i.e. closure hierarchies of orbits and bundles, see the examples in [7, 9, 20]. These stratifications are illustrated by the graphs showing all canonical forms that the symmetric matrix pencils may have in arbitrarily small neighbourhoods of a given symmetric matrix pencil. For example, the stratifications show how a Jordan-like block can split into two Jordan-like blocks associated with two different eigenvalues. The stratification graphs are known for matrices [19], general matrix pencils [19], matrix pencils associated with state-space systems [13], matrix polynomials [14, 25], as well as for the skew-symmetric matrix pencils [15] and polynomials [8]. Nevertheless the stratification theory for symmetric matrix pencils remains to be an open and challenging problem and this paper can be seen as a step 
towards a better understanding of small perturbations of symmetric matrix pencils and thus towards a development of the stratification theory.

This paper and the paper [7] are directed towards the same audience. To facilitate the reading and the use of results from [7], we keep their style, structure, notation as similar as possible, and organize the rest of this paper as follows. In Section 2, we start by recalling some preliminary information needed to present the miniversal deformations of symmetric matrix pencils. We also give an upper bound on the distance between a symmetric matrix pencil and its miniversal deformation as well as compute the codimensions of the congruence orbits of symmetric matrix pencils. (The Matlab functions for computing these codimensions were developed [12] and became a part of the Matrix Canonical Structure (MCS) Toolbox [24].) In Section 3.1 we present a method for constructing the miniversal deformations. In the remaining parts of Section 3 we derive the deformations step by step, namely, for the diagonal blocks in Section 3.2, for the off-diagonal blocks that correspond to the canonical summands of the same type in Section 3.3, and finally, for the off-diagonal blocks that correspond to the canonical summands of different types in Section 3.4.

In this paper all matrices are considered over the field of complex numbers. To refer to a matrix pair, we use calligraphic letters, e.g., $\mathcal{A}$ or $\mathcal{D}$.

\section{Miniversal deformations of pairs of symmetric matrices}

We start this section by recalling the canonical form of pairs of symmetric matrices under congruence given in [32], then we present some preliminaries on miniversal deformations followed by our main theorems. The miniversal deformations derived in Theorem 2.1 will be proven in Section 3 .

For each $n=1,2, \ldots$, define the $n \times n$ matrices

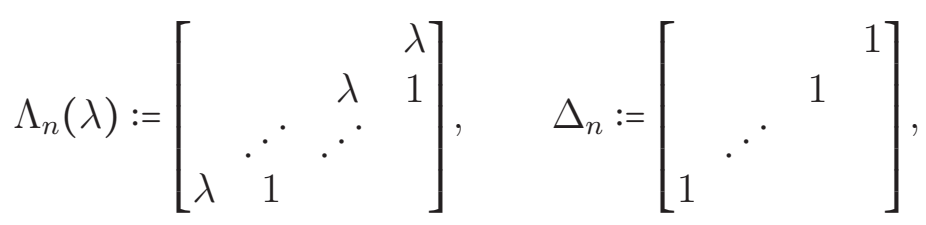

where $\lambda \in \mathbb{C}$, and the $n \times(n+1)$ matrices

$$
F_{n}:=\left[\begin{array}{cccc}
1 & 0 & & \\
& \ddots & \ddots & \\
& & 1 & 0
\end{array}\right], \quad G_{n}:=\left[\begin{array}{cccc}
0 & 1 & & \\
& \ddots & \ddots & \\
& & 0 & 1
\end{array}\right] .
$$

All non-specified entries of the matrices $\Lambda_{n}(\lambda), \Delta_{n}, F_{n}$, and $G_{n}$ are zeros. 
Lemma 2.1 ([32]). Every pair of symmetric complex matrices is congruent to a direct sum

$$
(A, B)_{\mathrm{can}}=\bigoplus_{i=1}^{a} \mathcal{H}_{h_{i}}\left(\lambda_{i}\right) \oplus \bigoplus_{j=1}^{b} \mathcal{K}_{k_{j}} \oplus \bigoplus_{r=1}^{c} \mathcal{L}_{l_{r}}
$$

where

$$
\begin{aligned}
\mathcal{H}_{n}(\lambda) & :=\left(\Delta_{n}, \Lambda_{n}(\lambda)\right), \quad \lambda \in \mathbb{C} \\
\mathcal{K}_{n} & :=\left(\Lambda_{n}(0), \Delta_{n}\right), \\
\mathcal{L}_{n} & :=\left(\left[\begin{array}{cc}
0 & F_{n}^{T} \\
F_{n} & 0
\end{array}\right],\left[\begin{array}{cc}
0 & G_{n}^{T} \\
G_{n} & 0
\end{array}\right]\right) .
\end{aligned}
$$

The sum (11) is determined uniquely up to permutation of summands.

We extend Arnold's concept of miniversal deformations to pairs of symmetric matrices with respect to congruence in the same manner as it was done for pairs of skew-symmetric matrices [7]. Similarly miniversal deformations has been defined for matrix pencils [18, 22], as well as for matrices of bilinear [10] and sesquilinear 11] forms.

A deformation of a pair of symmetric $\hat{n} \times \hat{n}$ matrices $(A, B)$ is a holomorphic mapping $\mathcal{A}(\vec{\delta})$, where $\vec{\delta}=\left(\delta_{1}, \ldots, \delta_{k}\right)$, from a neighborhood $\Omega \subset \mathbb{C}^{k}$ of $\overrightarrow{0}=(0, \ldots, 0)$ to the space of pairs of symmetric $\hat{n} \times \hat{n}$ matrices such that $\mathcal{A}(\overrightarrow{0})=(A, B)$. In this paper we consider only symmetric deformations, i.e. we preserve the symmetric structure of matrix pairs. Thus, with no risk of confusion, we write "deformation" but not "symmetric deformation".

Definition 2.1. A deformation $\mathcal{A}\left(\delta_{1}, \ldots, \delta_{k}\right)$ of a pair of symmetric matrices $(A, B)$ is called versal if for every deformation $\mathcal{B}\left(\sigma_{1}, \ldots, \sigma_{l}\right)$ of $(A, B)$ we have

$$
\mathcal{B}\left(\sigma_{1}, \ldots, \sigma_{l}\right)=I\left(\sigma_{1}, \ldots, \sigma_{l}\right)^{T} \mathcal{A}\left(\varphi_{1}(\vec{\sigma}), \ldots, \varphi_{k}(\vec{\sigma})\right) I\left(\sigma_{1}, \ldots, \sigma_{l}\right),
$$

where $I\left(\sigma_{1}, \ldots, \sigma_{l}\right)$ is a deformation of the identity matrix, and all $\varphi_{i}(\vec{\sigma})$ are convergent in a neighborhood of $\overrightarrow{0}$ power series such that $\varphi_{i}(\overrightarrow{0})=0$. A versal deformation $\mathcal{A}\left(\delta_{1}, \ldots, \delta_{k}\right)$ of $(A, B)$ is called miniversal if there is no versal deformation having less than $k$ parameters.

Define a $(0, *)$ matrix to be a matrix whose entries are 0 and $*$. A pair of symmetric matrices is of the form $\mathcal{D}$, where $\mathcal{D}$ is a pair of $(0, *)$ symmetric matrices, if it can be obtained from $\mathcal{D}$ by replacing the stars with complex numbers, respecting the symmetry. By $\mathcal{D}(\mathbb{C})$ we denote the space of all pairs of symmetric matrices of the form $\mathcal{D}$, and by $\mathcal{D}(\vec{\varepsilon})$ we denote the pair of parametric symmetric matrices obtained from $\mathcal{D}$ by replacing the $(i, j)$ th 
and $(j, i)$ th stars, $i \leq j$, with the parameter $\varepsilon_{i j}$ in the first matrix, and the $\left(i^{\prime}, j^{\prime}\right)$ th and $\left(j^{\prime}, i^{\prime}\right)$ th stars, $i^{\prime} \leq j^{\prime}$, with the parameter $\varepsilon_{i^{\prime} j^{\prime}}^{\prime}$ in the second matrix. That is to say

$$
\begin{gathered}
\mathcal{D}(\vec{\varepsilon}):=\left(\sum_{(i, j) \in \operatorname{Ind}_{1}(\mathcal{D})} \varepsilon_{i j} E_{i j}, \sum_{\left(i^{\prime}, j^{\prime}\right) \in \operatorname{Ind}_{2}(\mathcal{D})} \varepsilon_{i^{\prime} j^{\prime}}^{\prime} E_{i^{\prime} j^{\prime}}\right), \\
\mathcal{D}(\mathbb{C}):=\left\{\mathcal{D}(\vec{\varepsilon}) \mid \vec{\varepsilon} \in \mathbb{C}^{k}\right\}=\left\{\left(\underset{(i, j) \in \operatorname{Ind}_{1}(\mathcal{D})}{+} \mathbb{C} E_{i j}, \underset{\left(i^{\prime}, j^{\prime}\right) \in \operatorname{Ind}_{2}(\mathcal{D})}{+} \mathbb{C} E_{i^{\prime} j^{\prime}}\right)\right\},
\end{gathered}
$$

where each $E_{i j}$ is the matrix whose $(i, j)$ th and $(j, i)$ th entries are 1 , and the other entries are zero, "+" denotes the entrywise sum of matrices, and $\operatorname{Ind}_{1}(\mathcal{D}), \operatorname{Ind}_{2}(\mathcal{D}) \subseteq\{1, \ldots, \hat{n}\} \times\{1, \ldots, \hat{n}\}$, are the sets of indices of the stars in the upper-triangular parts (including the main diagonals) of the first and the second matrices of the pair $\mathcal{D}$. A miniversal deformation of $(A, B)$ is simplest if it has the form $(A, B)+\mathcal{D}(\vec{\varepsilon})$, where $\mathcal{D}$ is a pair of $(0, *)$ matrices, see also [7, 10, 11, 22].

In other words, for all pairs of $\hat{n} \times \hat{n}$ symmetric matrices $\left(A+E, B+E^{\prime}\right)$ that are close to a given pair of symmetric matrices $(A, B)$, we derive the normal form $\mathcal{A}\left(E, E^{\prime}\right)$ with respect to the congruence transformation

$$
\left(A+E, B+E^{\prime}\right) \mapsto S\left(E, E^{\prime}\right)^{T}\left(A+E, B+E^{\prime}\right) S\left(E, E^{\prime}\right)=: \mathcal{A}\left(E, E^{\prime}\right)
$$

in which $S\left(E, E^{\prime}\right)$ is nonsingular and holomorphic at 0 (i.e. its entries are power series in the entries of $E$ and $E^{\prime}$ that are convergent in a neighborhood of 0$)$.

We define $\mathcal{A}(0,0)$ to be equal to the congruence canonical form $(A, B)_{\mathrm{can}}$, see (11), of $(A, B)$. Then

$$
\mathcal{A}\left(E, E^{\prime}\right)=(A, B)_{\mathrm{can}}+\mathcal{D}\left(E, E^{\prime}\right),
$$

where $\mathcal{D}\left(E, E^{\prime}\right)\left(\mathcal{D}\left(E, E^{\prime}\right)=\mathcal{D}(\vec{\varepsilon})\right.$ for some $\left.\vec{\varepsilon} \in \mathbb{C}^{k}\right)$ is a pair of symmetric matrices that is holomorphic at 0 and $\mathcal{D}(0,0)=(0,0)$. In Theorem 2.1 we present $\mathcal{D}\left(E, E^{\prime}\right)$ with the minimal number of nonzero entries that is attainable using the congruence transformation (7).

Define the following $(0, *)$ matrices, where each star denotes a holomorphic at zero function of the entries of $E$ and $E^{\prime}$ :

- $0_{m n}$ is the $m \times n$ zero matrix;

- $0_{m n *}$ is the $m \times n$ matrix $\left[\begin{array}{ccc} & & 0 \\ 0_{m-1, n-1} & \vdots \\ 0 & \ldots & 0\end{array}\right]$; 
- $0_{m n}^{\leftarrow}\left(\right.$ resp. $\left.0_{m n}^{\rightarrow}\right)$ is the $m \times n$ matrix $\left[\begin{array}{cc}* & \\ \vdots & 0_{m, n-1} \\ * & \end{array}\right]\left(\operatorname{resp.}\left[\begin{array}{cc} & * \\ 0_{m, n-1} & \vdots \\ & *\end{array}\right]\right)$;

- $0_{m n}^{\nwarrow}$ is the $m \times n$ matrix

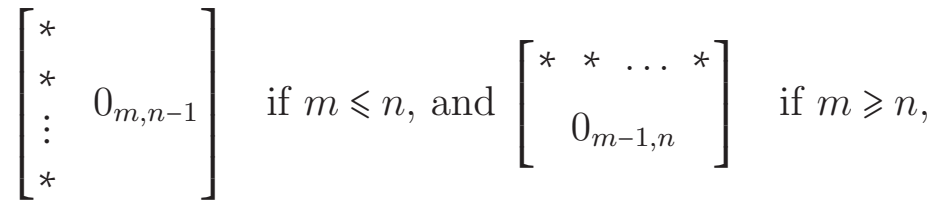

if $m=n$, then we can choose any of the matrices defined in (9);

- $0_{m n}$ is the $m \times n$ matrix $\left[\begin{array}{ccc}* & \ldots & * \\ & 0_{m-1, n-1} & \vdots \\ & & *\end{array}\right]$ or $\left[\begin{array}{ccc}* & & \\ \vdots & 0_{m-1, n-1} & \\ * & \ldots & *\end{array}\right]$;

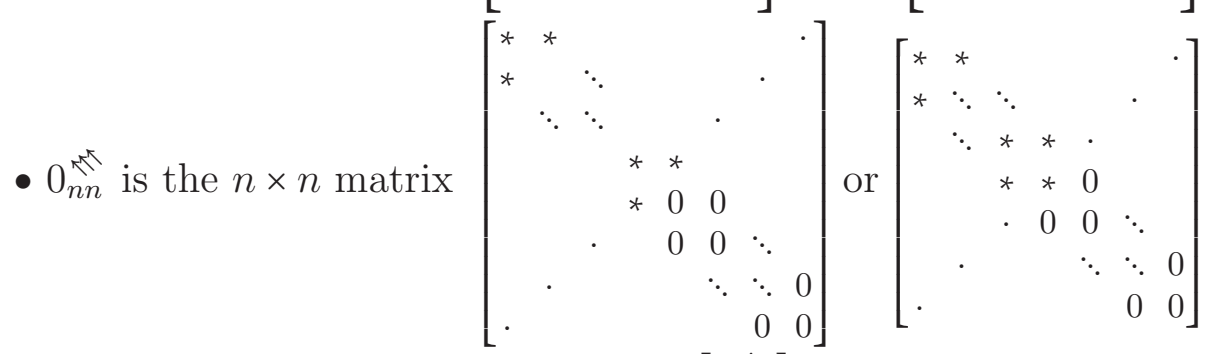

for $n$ being even or, respectively, odd; $\lceil n / 2\rceil$ stars on the main diagonal and $\lfloor n / 2\rfloor$ stars on each of the sub- and overdiagonals;

- $0_{n n}$ is is the $n \times n$ matrix $\left[\begin{array}{cccc}* & * & & \\ * & * & \ddots & \\ & \ddots & \ddots & * \\ & * & *\end{array}\right]$;

- $0_{m n}^{\boxminus}$ with $m<n$ is the $m \times n$ matrix

$$
\left[\begin{array}{cccc}
0 & \ldots & 0 & \\
\vdots & & \vdots & 0_{n-1, n-m+1} \\
0 & \ldots & 0 & * \ldots * 0
\end{array}\right] \quad(n-m \text { stars })
$$

if $m \geq n$ then $0_{m n}^{\boxminus}=0$.

If there is no risk of confusion, we will omit the indices $m$ and $n$.

Consider a canonical pair of symmetric matrices under congruence

$$
(A, B)_{\mathrm{can}}=\mathcal{X}_{1} \oplus \cdots \oplus \mathcal{X}_{t}
$$

where $\mathcal{X}_{1}, \ldots, \mathcal{X}_{t}$ are pairs of the form (2)-(41), and let $\mathcal{D}\left(E, E^{\prime}\right)$ be a pair of symmetric matrices, defined in (86), whose matrices are partitioned into 
blocks conformally to the decomposition (10), i.e.

$\mathcal{D}\left(E, E^{\prime}\right)=\mathcal{D}=\left(\left[\begin{array}{ccc}D_{11} & \ldots & D_{1 t} \\ \vdots & \ddots & \vdots \\ D_{t 1} & \ldots & D_{t t}\end{array}\right],\left[\begin{array}{ccc}D_{11}^{\prime} & \ldots & D_{1 t}^{\prime} \\ \vdots & \ddots & \vdots \\ D_{t 1}^{\prime} & \ldots & D_{t t}^{\prime}\end{array}\right]\right),\left(D_{j i}, D_{j i}^{\prime}\right)=\left(D_{i j}^{T}, D_{i j}^{\prime T}\right)$.

Define

$$
\mathcal{D}\left(\mathcal{X}_{i}\right):=\left(D_{i i}, D_{i i}^{\prime}\right) \quad \text { and } \quad \mathcal{D}\left(\mathcal{X}_{i}, \mathcal{X}_{j}\right):=\left(D_{i j}, D_{i j}^{\prime}\right), i<j .
$$

It is sufficient to construct miniversal deformations for all canonical pairs of matrices; i.e., for all direct sums of the pairs (2)-(44), since each pair of symmetric matrices is congruent to its canonical form, see Lemma 2.1.

Theorem 2.1. Let $(A, B)_{\text {can }}$ be a canonical pair of symmetric complex matrices (1). A simplest miniversal deformation of $(A, B)_{\text {can }}$ can be taken in the form $(A, B)_{\mathrm{can}}+\mathcal{D}$ in which $\mathcal{D}$ is a pair of $(0, *)$ matrices (the stars denote independent parameters, up to symmetry, see also Remark 2.1) whose matrices are partitioned into blocks conformally to the decomposition of $(A, B)_{\mathrm{can}}$, see (11), and the blocks of $\mathcal{D}$ are defined, in the notation (12), as follows:

(i) The diagonal blocks of $\mathcal{D}$ are defined by

$$
\begin{aligned}
& \mathcal{D}\left(\mathcal{H}_{n}(\lambda)\right)=\left(0,0^{\text {자 }}\right), \\
& \mathcal{D}\left(\mathcal{K}_{n}\right)=\left(0^{\text {차 }}, 0\right), \\
& \mathcal{D}\left(\mathcal{L}_{n}\right)=\left(\left[\begin{array}{cc}
0_{*} & 0 \\
0 & 0
\end{array}\right],\left[\begin{array}{cc}
0 & 0 \\
0 & 0
\end{array}\right]\right) .
\end{aligned}
$$

(ii) The off-diagonal blocks of $\mathcal{D}$ whose horizontal and vertical strips contain summands of $(A, B)_{\text {can }}$ of the same type are defined by

$$
\begin{aligned}
\mathcal{D}\left(\mathcal{H}_{n}(\lambda), \mathcal{H}_{m}(\mu)\right) & = \begin{cases}(0,0) & \text { if } \lambda \neq \mu, \\
\left(0,0^{\star}\right) & \text { if } \lambda=\mu,\end{cases} \\
\mathcal{D}\left(\mathcal{K}_{n}, \mathcal{K}_{m}\right) & =\left(0^{\star}, 0\right), \\
\mathcal{D}\left(\mathcal{L}_{n}, \mathcal{L}_{m}\right) & =\left(\left[\begin{array}{cc}
0_{*} & 0 \\
0 & 0
\end{array}\right],\left[\begin{array}{cc}
0\urcorner & 0_{n+1, m}^{\boxminus} \\
0_{m+1, n}^{\boxminus T} & 0
\end{array}\right]\right) .
\end{aligned}
$$

(iii) The off-diagonal blocks of $\mathcal{D}$ whose horizontal and vertical strips contain summands of $(A, B)_{\mathrm{can}}$ of different types are defined by:

$$
\begin{aligned}
\mathcal{D}\left(\mathcal{H}_{n}(\lambda), \mathcal{K}_{m}\right) & =(0,0), \\
\mathcal{D}\left(\mathcal{H}_{n}(\lambda), \mathcal{L}_{m}\right) & =\left(0,0^{\leftarrow}\right), \\
\mathcal{D}\left(\mathcal{K}_{n}, \mathcal{L}_{m}\right) & =\left(\left[\begin{array}{ll}
0 \rightarrow & 0
\end{array}\right], 0\right) .
\end{aligned}
$$


Remark 2.1 (Independency of parameters). All parameters that are placed instead of the stars in the upper triangular parts, including the main diagonals, of matrices of $\mathcal{D}$ are independent and the strictly lower triangular parts of matrices of $\mathcal{D}$ are defined by the symmetry. For example, it means that parametric matrix pairs obtained from $\left(D_{i j}, D_{i j}^{\prime}\right)$ and $\left(D_{i^{\prime} j^{\prime}}, D_{i^{\prime} j^{\prime}}^{\prime}\right)$ have dependent (actually, equal) parameter entries if and only if $i^{\prime}=j$ and $j^{\prime}=i$.

Now we explain how the matrix pair $\mathcal{D}$ defined in (11) is constructed in Section 3. The vector space

$$
T_{(A, B)}:=\left\{C^{T}(A, B)_{\mathrm{can}}+(A, B)_{\mathrm{can}} C \mid C \in \mathbb{C}^{\hat{n} \times \hat{n}}\right\}
$$

is the tangent space to the congruence class of $(A, B)_{\text {can }}$ at the point $(A, B)_{\text {can }}$. Then $\mathcal{D}$ satisfies the following condition:

$$
\mathbb{C}_{s}^{\hat{n} \times \hat{n}} \times \mathbb{C}_{s}^{\hat{n} \times \hat{n}}=T_{(A, B)}+\mathcal{D}(\mathbb{C})
$$

where $\mathbb{C}_{s}^{\hat{n} \times \hat{n}}$ is the space of all $\hat{n} \times \hat{n}$ symmetric matrices and $\mathcal{D}(\mathbb{C})$, defined in (6), is the vector space of all matrix pairs obtained from $\mathcal{D}$ by replacing its stars by complex numbers. Thus, the number of stars in the uppertriangular part of $\mathcal{D}$ (including the main diagonal) is equal to the codimension of the congruence class of $(A, B)_{\text {can }}$. Recall that the codimension of congruence orbit of $(A, B)$ is defined as the dimension of the normal space $N_{(A, B)}$ at the point $(A, B)$ and $N_{(A, B)}$ is the orthogonal complement to the tangent space $T_{(A, B)}$ in $\mathbb{C}_{s}^{\hat{n} \times \hat{n}} \times \mathbb{C}_{s}^{\hat{n} \times \hat{n}}$.

Following [7] and using the norm of the original perturbations, we bound the distance from the miniversal deformations of a symmetric matrix pair to this matrix pair. Notably, this distance can be made arbitrarily small by decreasing the size of the allowed perturbations.

By (22), for each pair of symmetric $\hat{n}$-by- $\hat{n}$ matrices $\left(E_{i j}, 0\right)$ and $\left(0, E_{i^{\prime} j^{\prime}}\right)$, $1 \leqslant i, j, i^{\prime}, j^{\prime} \leqslant \hat{n}$ there exist $X_{i j}, X_{i^{\prime} j^{\prime}}^{\prime} \in \mathbb{C}^{\hat{n} \times \hat{n}}$ such that

$$
\begin{gathered}
\left(E_{i j}, 0\right)+X_{i j}^{T}(A, B)+(A, B) X_{i j} \in \mathcal{D}(\mathbb{C}), \\
\left(0, E_{i^{\prime} j^{\prime}}\right)+X_{i^{\prime} j^{\prime}}^{\prime T}(A, B)+(A, B) X_{i^{\prime} j^{\prime}}^{\prime} \in \mathcal{D}(\mathbb{C}),
\end{gathered}
$$

where $\mathcal{D}(\mathbb{C})$ is defined in $\left([6)\right.$. If $(i, j) \in \operatorname{Ind}_{1}(\mathcal{D})$, then $\left(E_{i j}, 0\right) \in \mathcal{D}(\mathbb{C})$, and so we can put $X_{i j}=0$. Analogously, if $\left(i^{\prime}, j^{\prime}\right) \in \operatorname{Ind}_{2}(\mathcal{D})$, then $\left(0, E_{i^{\prime} j^{\prime}}\right) \in \mathcal{D}(\mathbb{C})$, and so we can put $X_{i^{\prime} j^{\prime}}=0$. Denote

$$
\gamma:=\sum_{(i, j) \notin \operatorname{Ind}_{1}(\mathcal{D})}\left\|X_{i j}\right\|+\sum_{\left(i^{\prime}, j^{\prime}\right) \notin \operatorname{Ind}_{2}(\mathcal{D})}\left\|X_{i^{\prime} j^{\prime}}^{\prime}\right\|,
$$

where $\|\cdot\|$ denotes the Frobenius norm. 
Theorem 2.2 (Upper bound for the norm of miniversal deformations). Let $(A, B) \in\left(\mathbb{C}_{s}^{\hat{n} \times \hat{n}}, \mathbb{C}_{s}^{\hat{n} \times \hat{n}}\right)$ and let $\varepsilon \in \mathbb{R}$ such that $0<\varepsilon<\kappa$ where $\kappa=(\max \{1+$ $\gamma(\alpha+1)(2+\gamma), 1+\gamma(\beta+1)(2+\gamma)\})^{-1}$ with $\alpha:=\|A\|, \beta:=\|B\|$ and $\gamma$ is defined in (23). For each pair of symmetric $\hat{n}$-by- $\hat{n}$ matrices $(M, N)$ satisfying

$$
\|M\|<\varepsilon^{2}, \quad\|N\|<\varepsilon^{2},
$$

there exists a matrix $S=I_{\hat{n}}+X$ depending holomorphically on the entries of $(M, N)$ in a neighborhood of zero such that

$S^{T}(A+M, B+N) S=(A+P, B+Q), \quad(P, Q) \in \mathcal{D}(\mathbb{C}),\|P\|<\varepsilon$, and $\|Q\|<\varepsilon$, where $\mathbb{C}_{s}^{\hat{n} \times \hat{n}} \times \mathbb{C}_{s}^{\hat{n} \times \hat{n}}=T_{(A, B)_{\text {can }}}+\mathcal{D}(\mathbb{C})$.

The proof of Theorem 2.2 is analogous to the proof of Theorem 2.2 in [7] and we omit it.

In the following corollary we compute explicitly the codimensions of orbits of pairs of symmetric complex matrices. Alternatively, these codimensions can be computed using Corollary 2.2 and Theorem 2.3 of [16].

Corollary 2.1. The codimension of congruence orbit of a pair of symmetric complex matrices in the congruence canonical form $(A, B)_{\text {can }}=\bigoplus_{i=1}^{a} \mathcal{H}_{h_{i}}\left(\lambda_{i}\right) \oplus$ $\bigoplus_{j=1}^{b} \mathcal{K}_{k_{j}} \oplus \bigoplus_{r=1}^{c} \mathcal{L}_{l_{r}}$ can be computed as follows:

$$
\operatorname{codim}(A, B)_{\text {can }}=c_{\mathcal{H}}+c_{\mathcal{K}}+c_{\mathcal{L}}+c_{\mathcal{H} H}+c_{\mathcal{K K}}+c_{\mathcal{L L}}+c_{\mathcal{H} K}+c_{\mathcal{H L}}+c_{\mathcal{K} \mathcal{L}},
$$

where the summands correspond to

- the direct summands of (10) of the same type:

$$
c_{\mathcal{H}}:=\sum_{i=1}^{a} h_{i}, \quad c_{\mathcal{K}}:=\sum_{j=1}^{b} k_{j}, \quad c_{\mathcal{L}}:=2 \sum_{r=1}^{c}\left(l_{r}+1\right)
$$

- the pairs of direct summands of (11) of the same type:

$$
\begin{gathered}
c_{\mathcal{H} \mathcal{H}}:=\sum_{\substack{i<i^{\prime} \\
\lambda_{i}=\lambda_{i^{\prime}}}} \min \left(h_{i}, h_{i^{\prime}}\right), \quad c_{\mathcal{K} \mathcal{K}}:=\sum_{j<j^{\prime}} \min \left(k_{j}, k_{j^{\prime}}\right), \\
c_{\mathcal{L} \mathcal{L}}:=\sum_{r<r^{\prime}}\left(2 \max \left(l_{r}, l_{r^{\prime}}\right)+\varepsilon_{r r^{\prime}}\right), \quad \text { in which } \varepsilon_{r r^{\prime}}:= \begin{cases}2 & \text { if } l_{r}=l_{r^{\prime}}, \\
1 & \text { if } l_{r} \neq l_{r^{\prime}}\end{cases}
\end{gathered}
$$

- the pairs of direct summands of (11) of different types:

$$
c_{\mathcal{H} \mathcal{K}}:=0, \quad c_{\mathcal{H} \mathcal{L}}:=c \sum_{i} h_{i}, \quad c_{\mathcal{K} \mathcal{L}}:=c \sum_{j} k_{j} .
$$

Proof. The numbers $c_{\mathcal{H}}, c_{\mathcal{K}}, \ldots, c_{\mathcal{K} L}$ are obtained by counting the independent parameters in the miniversal deformations from Theorem 2.1, 


\section{Proof of Theorem 2.1}

\subsection{Construction of miniversal deformations}

To make this paper self-contained, we briefly describe how to construct the simplest miniversal deformations. This method is presented with more details in [7, 10, 11] and will be used to prove Theorem 2.1.

For a matrix pair $(A, B)$, the deformation

$$
\mathcal{U}(\vec{\varepsilon}):=\left(A+\sum_{i=1}^{\hat{n}} \sum_{j=i}^{\hat{n}} \varepsilon_{i j} E_{i j}, B+\sum_{i=1}^{\hat{n}} \sum_{j=i}^{\hat{n}} \varepsilon_{i j}^{\prime} E_{i j}\right)
$$

is universal in the following sense: every deformation $\mathcal{B}\left(\mu_{1}, \ldots, \mu_{l}\right)$ of $(A, B)$ has the form $\mathcal{U}\left(\vec{\varphi}\left(\mu_{1}, \ldots, \mu_{l}\right)\right)$, where $\varphi_{i j}\left(\mu_{1}, \ldots, \mu_{l}\right)$ are convergent in a neighborhood of $\overrightarrow{0}$ power series such that $\varphi_{i j}(\overrightarrow{0})=0$. Hence "every deformation $\mathcal{B}\left(\mu_{1}, \ldots, \mu_{l}\right)$ " in Definition 2.1 can be replaced by $\mathcal{U}(\vec{\varepsilon})$.

The following lemma ensures that any matrix pair with entries 0 and * that satisfies (22) can be taken as a versal deformation of $(A, B)$. For the proof of Lemma 3.1 see [7, Lemma 3.2]. Recall that, for a subspace $U$ of a vector space $V$, a coset of $U$ in $V$ is a set $v+U$, where $v \in V$.

Lemma 3.1. Let $(A, B) \in\left(\mathbb{C}_{s}^{\hat{n} \times \hat{n}}, \mathbb{C}_{s}^{\hat{n} \times \hat{n}}\right)$ and let $\mathcal{D}$ be a pair of $(0, *)$ matrices of size $\hat{n} \times \hat{n}$. The following are equivalent:

(i) The deformation $(A, B)+\mathcal{D}(\vec{\varepsilon})$ defined in (5) is miniversal.

(ii) The vector space $\left(\mathbb{C}_{s}^{\hat{n} \times \hat{n}}, \mathbb{C}_{s}^{\hat{n} \times \hat{n}}\right)$ decomposes into the direct sum

$$
\left(\mathbb{C}_{s}^{\hat{n} \times \hat{n}}, \mathbb{C}_{s}^{\hat{n} \times \hat{n}}\right)=T_{(A, B)}+\mathcal{D}(\mathbb{C}), \quad T_{(A, B)} \cap \mathcal{D}(\mathbb{C})=\{(A, B)\} .
$$

(iii) Each coset of $T_{(A, B)}$ in $\left(\mathbb{C}_{s}^{\hat{n} \times \hat{n}}, \mathbb{C}_{s}^{\hat{n} \times \hat{n}}\right)$ contains exactly one matrix of the form $\mathcal{D}$.

Recall that versality of each deformation $(A, B)+\mathcal{D}(\vec{\varepsilon})$ in which $\mathcal{D}$ satisfies (27) means that there exists a deformation $\mathcal{I}(\vec{\varepsilon})$ of the identity matrix such that $\mathcal{D}(\vec{\varepsilon})=\mathcal{I}(\vec{\varepsilon})^{T} \mathcal{U}(\vec{\varepsilon}) \mathcal{I}(\vec{\varepsilon})$, where $\mathcal{U}(\vec{\varepsilon})$ is defined in (26) .

A simplest miniversal deformation of $(A, B) \in\left(\mathbb{C}_{s}^{\hat{n} \times \hat{n}}, \mathbb{C}_{s}^{\hat{n} \times \hat{n}}\right)$ can be constructed as follows. Let $\left(E_{1}, \ldots, E_{\hat{n}(\hat{n}+1)}\right)$ be the basis of $\left(\mathbb{C}_{s}^{\hat{n} \times \hat{n}}, \mathbb{C}_{s}^{\hat{n} \times \hat{n}}\right)$ in which every $E_{k}$ is either of the form $\left(E_{i j}, 0\right)$ or $\left(0, E_{i^{\prime} j^{\prime}}\right)$ and let $\left(T_{1}, \ldots, T_{r}\right)$ be a basis of the space $T_{(A, B)}$. By removing from the sequence $\left(T_{1}, \ldots, T_{r}, E_{1}, \ldots, E_{\hat{n}(\hat{n}+1)}\right)$ every pair of matrices that is a linear combination 
of the preceding matrices, we receive a new basis $\left(T_{1}, \ldots, T_{r}, E_{i_{1}}, \ldots, E_{i_{k}}\right)$ of the space $\left(\mathbb{C}_{s}^{\hat{n} \times \hat{n}}, \mathbb{C}_{s}^{\hat{n} \times \hat{n}}\right)$. By Lemma 3.1, the deformation

$$
\begin{aligned}
\mathcal{A}\left(\varepsilon_{1}, \ldots, \varepsilon_{k_{1}}, \varepsilon_{1}^{\prime}, \ldots, \varepsilon_{k_{2}}^{\prime}\right) & =(A, B)+\varepsilon_{1} E_{1}+\cdots+\varepsilon_{k_{1}} E_{i_{k_{1}}}+\varepsilon_{1}^{\prime} E_{i_{k_{1}+1}}+\cdots+\varepsilon_{k_{2}}^{\prime} E_{i_{k}} \\
& =(A, B)+\varepsilon_{1}\left(E_{i_{1}, j_{1}}, 0\right)+\cdots+\varepsilon_{k_{1}}\left(E_{i_{k_{1}} j_{k_{1}}}, 0\right) \\
& +\varepsilon_{1}^{\prime}\left(0, E_{i_{k_{1}+1}, j_{k_{1}+1}}\right)+\cdots+\varepsilon_{k_{2}}^{\prime}\left(0, E_{i_{k}, j_{k}}\right),
\end{aligned}
$$

where $k_{1}+k_{2}=k$, is miniversal.

For each pair of $m \times m$ symmetric matrices $\left(A_{1}, B_{1}\right)$ and each pair $n \times n$ symmetric matrices $\left(A_{2}, B_{2}\right)$, define the vector spaces

$$
\begin{aligned}
V\left(A_{1}, B_{1}\right):= & \left\{S^{T}\left(A_{1}, B_{1}\right)+\left(A_{1}, B_{1}\right) S, \text { where } S \in \mathbb{C}^{m \times m}\right\} . \\
V\left(\left(A_{1}, B_{1}\right),\left(A_{2}, B_{2}\right)\right) & :=\left\{\left(R^{T}\left(A_{2}, B_{2}\right)+\left(A_{1}, B_{1}\right) S, S^{T}\left(A_{1}, B_{1}\right)+\left(A_{2}, B_{2}\right) R\right),\right. \\
& \text { where } \left.S \in \mathbb{C}^{m \times n} \text { and } R \in \mathbb{C}^{n \times m}\right\} .
\end{aligned}
$$

Lemma 3.2. Let $(A, B)=\left(A_{1}, B_{1}\right) \oplus \cdots \oplus\left(A_{t}, B_{t}\right)$ be a block-diagonal matrix in which every $\left(A_{i}, B_{i}\right)$ is $n_{i} \times n_{i}$. Let $\mathcal{D}$ be a pair of $(0, *)$ matrices of the same size as $(A, B)$ and partitioned into blocks $\left(D_{i j}, D_{i j}^{\prime}\right)$ conformably to the partition of $(A, B)$, see (11). Then $(A, B)+\mathcal{D}\left(E, E^{\prime}\right)$ is a simplest miniversal deformation of $(A, B)$ for congruence if and only if

(i) every coset of $V\left(A_{i}, B_{i}\right)$ in $\left(\mathbb{C}_{s}^{n_{i} \times n_{i}}, \mathbb{C}_{s}^{n_{i} \times n_{i}}\right)$ contains exactly one matrix of the form $\left(D_{i i}, D_{i i}^{\prime}\right)$, and

(ii) every coset of $V\left(\left(A_{i}, B_{i}\right),\left(A_{i}^{T}, B_{i}^{T}\right)\right)$ in $\left(\mathbb{C}^{n_{i} \times n_{j}}, \mathbb{C}^{n_{i} \times n_{j}}\right) \oplus$ $\left(\mathbb{C}^{n_{j} \times n_{i}}, \mathbb{C}^{n_{j} \times n_{i}}\right)$ contains exactly two pairs of matrices $\left(\left(W_{1}, W_{2}\right),\left(W_{1}^{T}, W_{2}^{T}\right)\right)$, where $\left(W_{1}, W_{2}\right)$ is of the form $\left(D_{i j}, D_{i j}^{\prime}\right)$ and $\left(W_{1}^{T}, W_{2}^{T}\right)$ is of the form $\left(D_{j i}, D_{j i}^{\prime}\right)=\left(D_{i j}^{T}, D_{i j}^{\prime T}\right)$.

See [7, Lemma 3.3] for the proof of Lemma 3.2.

Corollary 3.1. By Lemma 3.2, $(A, B)+\mathcal{D}(\vec{\varepsilon})$ is a miniversal deformation of $(A, B)$ if and only if each submatrix of the form

$$
\left(\left[\begin{array}{cc}
A_{i}+D_{i i}(\vec{\varepsilon}) & D_{i j}(\vec{\varepsilon}) \\
D_{j i}(\vec{\varepsilon}) & A_{j}+D_{j j}(\vec{\varepsilon})
\end{array}\right],\left[\begin{array}{cc}
B_{i}+D_{i i}^{\prime}(\vec{\varepsilon}) & D_{i j}^{\prime}(\vec{\varepsilon}) \\
D_{j i}^{\prime}(\vec{\varepsilon}) & B_{j}+D_{j j}^{\prime}(\vec{\varepsilon})
\end{array}\right]\right), \quad i<j
$$

is a miniversal deformation of the pair $\left(A_{i} \oplus A_{j}, B_{i} \oplus B_{j}\right)$.

To prove Theorem 2.1, we show that the pairs (13)-(21) satisfy the conditions (i) and (ii) of Lemma 3.2. Each $\mathcal{X}_{i}$ in (10) is of the form $\mathcal{H}_{n}(\lambda)$, or $\mathcal{K}_{n}$, or $\mathcal{L}_{n}$, and so there are 9 types of pairs $\mathcal{D}\left(\mathcal{X}_{i}\right)$ and $\mathcal{D}\left(\mathcal{X}_{i}, \mathcal{X}_{j}\right)$ with $i<j$; they are given in (13)-(21). 


\subsection{Diagonal blocks of matrices of $\mathcal{D}$}

In Sections 3.2 .1 and 3.2 .2 we verify the condition (i) of Lemma 3.2 for the diagonal blocks of $\mathcal{D}$ defined in part (i) of Theorem 2.1.

\subsubsection{Diagonal blocks $\mathcal{D}\left(\mathcal{H}_{n}(\lambda)\right)$ and $\mathcal{D}\left(\mathcal{K}_{n}\right)$}

We start by considering the pair of blocks $\mathcal{H}_{n}(\lambda)$. Due to Lemma 3.2(i), it suffices to prove that each pair of symmetric $n$-by- $n$ matrices $(A, B)$ can be reduced to exactly one pair of matrices of the form (13) by adding

$$
\begin{aligned}
\delta(A, B)=(\delta A, \delta B) & =S^{T}\left(\Delta_{n}, \Lambda_{n}(\lambda)\right)+\left(\Delta_{n}, \Lambda_{n}(\lambda)\right) S \\
& =\left(S^{T} \Delta_{n}+\Delta_{n} S, S^{T} \Lambda_{n}(\lambda)+\Lambda_{n}(\lambda) S\right)
\end{aligned}
$$

in which $S$ is an arbitrary $n$-by- $n$ matrix. Obviously, that adding $S^{T} \Delta_{n}+\Delta_{n} S$ we reduce $A$ to zero. To preserve $A$, we must hereafter take $S$ such that $S^{T} \Delta_{n}+\Delta_{n} S=0$. This means that $S$ is skew symmetric with respect to its anti-diagonal. Therefore, we reduce $B$ by adding

$$
\begin{aligned}
& \delta B=S^{T} \Lambda_{n}(\lambda)+\Lambda_{n}(\lambda) S
\end{aligned}
$$

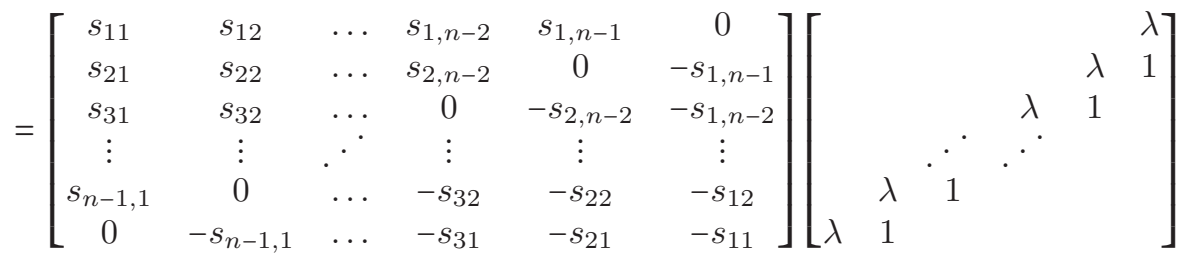

$$
\begin{aligned}
& +\left[\begin{array}{ccccccccc} 
& & & & \lambda \\
& & & \lambda & 1 \\
& & & \lambda & 1 & \\
& & \cdot & . & & \\
& \lambda & 1 & & & \\
\lambda & 1 & & & &
\end{array}\right]\left[\begin{array}{ccccccc}
s_{11} & s_{21} & \ldots & s_{n-2,1} & s_{n-1,1} & 0 \\
s_{12} & s_{22} & \ldots & s_{n-2,2} & 0 & -s_{n-1,1} \\
s_{13} & s_{23} & \ldots & 0 & -s_{n-2,2} & -s_{n-2,1} \\
\vdots & \vdots & . \cdot & \vdots & \vdots & \vdots \\
s_{1, n-1} & 0 & \ldots & -s_{23} & -s_{22} & -s_{21} \\
0 & -s_{1, n-1} & \ldots & -s_{13} & -s_{12} & -s_{11}
\end{array}\right] \\
& =\left[\begin{array}{ccccccc}
0 & 0 & s_{1, n-1} & s_{1, n-2} & \ldots & s_{13} & s_{12} \\
0 & -2 s_{1, n-1} & -s_{1, n-2} & s_{2, n-2}-s_{1, n-3} & \ldots & s_{23}-s_{12} & s_{22}-s_{11} \\
s_{1, n-1} & -s_{1, n-2} & -2 s_{2, n-2} & -s_{2, n-3} & \ldots & s_{33}-s_{22} & s_{32}-s_{21} \\
s_{1, n-2} & s_{2, n-2}-s_{1, n-3} & -s_{2, n-3} & -2 s_{3, n-3} & \ldots & s_{43}-s_{32} & s_{42}-s_{31} \\
\vdots & \vdots & \vdots & \vdots & \ddots & \vdots & \vdots \\
s_{13} & s_{23}-s_{12} & s_{33}-s_{22} & s_{43}-s_{32} & \ldots & -2 s_{n-2,2} & -s_{n-2,1} \\
s_{12} & s_{22}-s_{11} & s_{32}-s_{21} & s_{42}-s_{31} & \ldots & -s_{n-2,1} & -2 s_{n-1,1}
\end{array}\right]
\end{aligned}
$$

The upper part of each anti-diagonal of $\delta B$ has unique variables. Thus adding $\delta B$ we reduce each anti-diagonal of $B$ independently. We start from the upper left hand corner for each of the first $n$ anti-diagonals (note that two first anti-diagonals consist just of zeros); we have the following system 
of equations:

$$
\left[\begin{array}{cccc}
1 & & & \\
-1 & 1 & & \\
& \ddots & \ddots & \\
& & -1 & 1 \\
& & & -\alpha
\end{array}\right]\left[\begin{array}{c}
s_{-,-} \\
s_{-,-} \\
\vdots \\
s_{-,-}
\end{array}\right]=\left[\begin{array}{c}
b_{1} \\
b_{2} \\
\vdots \\
b_{t}
\end{array}\right],
$$

where $\alpha=2$ for odd anti-diagonals, $\alpha=1$ for even anti-diagonals, respectively, each $s_{-,-}$denotes the corresponding entry of (30) (we skip writing the subindices explicitly and only write "-, -", it will allow us to refer to (31) in following sections), and each $b_{i}$ denotes the corresponding entry of $B$. The matrix of the system (31) has $t-1$ columns and $t$ rows. By the KroneckerCapelli theorem, the system (31) does not have a solution, since the rank of (31) is equal to $(t-1)$ but the rank of the extended matrix of the system is $t$. Nevertheless, if we turn down the first or the last equation of the system (i.e. we do not set the first or the last element of the corresponding anti-diagonal of $B_{21}$ to zero), then (31) will have a solution. We chose to turn down the last equation to set to zero more elements (on odd anti-diagonals). For the remaining $(n-1)$ anti-diagonals we have a system of equations like (31) without the first equation, which has a solution. Therefore we can reduce the matrix $B$ to (13).

The result does not depend on $\lambda$ therefore $\mathcal{D}\left(\mathcal{H}_{n}(\lambda)\right)=\left(0,0^{\text {rर }}\right)$ and $\mathcal{D}\left(\mathcal{K}_{n}\right)=\left(0^{\text {kN }}, 0\right)$.

\subsubsection{Diagonal blocks $\mathcal{D}\left(\mathcal{L}_{n}\right)$}

Similarly to Section 3.2.1, i.e. using Lemma 3.2(i), we prove that each pair $(A, B)=\left(\left[\begin{array}{ll}A_{11} & A_{12} \\ A_{21} & A_{22}\end{array}\right],\left[\begin{array}{ll}B_{11} & B_{12} \\ B_{21} & B_{22}\end{array}\right]\right)$ of symmetric $(2 n+1)$-by- $(2 n+1)$ matrices can be reduced to (15) by adding

$$
\begin{aligned}
& \delta(A, B)=\left(\left[\begin{array}{ll}
\delta A_{11} & \delta A_{12} \\
\delta A_{21} & \delta A_{22}
\end{array}\right],\left[\begin{array}{ll}
\delta B_{11} & \delta B_{12} \\
\delta B_{21} & \delta B_{22}
\end{array}\right]\right) \\
& =\left[\begin{array}{cc}
S_{11}^{T} & S_{21}^{T} \\
S_{12}^{T} & S_{22}^{T}
\end{array}\right]\left(\left[\begin{array}{cc}
0 & F_{n}^{T} \\
F_{n} & 0
\end{array}\right],\left[\begin{array}{cc}
0 & G_{n}^{T} \\
G_{n} & 0
\end{array}\right]\right)+\left(\left[\begin{array}{cc}
0 & F_{n}^{T} \\
F_{n} & 0
\end{array}\right],\left[\begin{array}{cc}
0 & G_{n}^{T} \\
G_{n} & 0
\end{array}\right]\right)\left[\begin{array}{cc}
S_{11} & S_{12} \\
S_{21} & S_{22}
\end{array}\right] \\
& =\left(\left[\begin{array}{cc}
S_{21}^{T} F_{n}+F_{n}^{T} S_{21} & S_{11}^{T} F_{n}^{T}+F_{n}^{T} S_{22} \\
S_{22}^{T} F_{n}+F_{n} S_{11} & S_{12}^{T} F_{n}^{T}+F_{n} S_{12}
\end{array}\right],\left[\begin{array}{cc}
S_{21}^{T} G_{n}+G_{n}^{T} S_{21} & S_{11}^{T} G_{n}^{T}+G_{n}^{T} S_{22} \\
S_{22}^{T} G_{n}+G_{n} S_{11} & S_{12}^{T} G_{n}^{T}+G_{n} S_{12}
\end{array}\right]\right),
\end{aligned}
$$

where $S=\left[S_{i j}\right]_{i, j=1}^{2}$ is an arbitrary $(2 n+1) \times(2 n+1)$ matrix. Notably, each pair of blocks $\left(A_{i j}, B_{i j}\right), i, j=1,2$ of $(A, B)$ is changed independently. The pair of blocks $\left(A_{11}, B_{11}\right)$ is changed by adding $\left(S_{21}^{T} F_{n}+F_{n}^{T} S_{21}, S_{21}^{T} G_{n}+G_{n}^{T} S_{21}\right)$. Adding $\delta A_{11}=S_{21}^{T} F_{n}+F_{n}^{T} S_{21}$ we reduce each $(n+1)$-by- $(n+1)$ symmetric 
matrix $A_{11}$ to $0_{*}$. To preserve $A_{11}$, we must hereafter take $S_{21}$ such that $F_{n}^{T} S_{21}=-S_{21}^{T} F_{n}$. Therefore

$$
S_{21}=\left[\begin{array}{cccccc}
0 & s_{12} & s_{13} & \ldots & s_{1 n} & 0 \\
-s_{12} & 0 & s_{23} & \ldots & s_{2 n} & 0 \\
-s_{13} & -s_{23} & 0 & \ldots & s_{3 n} & 0 \\
\vdots & \vdots & \vdots & \ddots & \vdots & \vdots \\
-s_{1 n} & -s_{2 n} & -s_{3 n} & \ldots & 0 & 0
\end{array}\right]
$$

i.e. $S_{21}$ without the last column is skew symmetric. Now we reduce $B_{11}$ by adding

$$
\begin{aligned}
& \delta B_{11}=\left[\begin{array}{ccccc}
0 & -s_{12} & -s_{13} & \ldots & -s_{1 n} \\
s_{12} & 0 & -s_{23} & \ldots & -s_{2 n} \\
s_{13} & s_{23} & 0 & \ldots & -s_{3 n} \\
\vdots & \vdots & \vdots & \ddots & \vdots \\
s_{1 n} & s_{2 n} & s_{3 n} & \ldots & 0 \\
0 & 0 & 0 & \ldots & 0
\end{array}\right]\left[\begin{array}{llll}
0 & 1 & & \\
& \ddots & \ddots & \\
& & 0 & 1
\end{array}\right] \\
& +\left[\begin{array}{ccc}
0 & & \\
1 & \ddots & \\
& \ddots & 0 \\
& & 1
\end{array}\right]\left[\begin{array}{cccccc}
0 & s_{12} & s_{13} & \ldots & s_{1 n} & 0 \\
-s_{12} & 0 & s_{23} & \ldots & s_{2 n} & 0 \\
-s_{13} & -s_{23} & 0 & \ldots & s_{3 n} & 0 \\
\vdots & \vdots & \vdots & \ddots & \vdots & \vdots \\
-s_{1 n} & -s_{2 n} & -s_{3 n} & \ldots & 0 & 0
\end{array}\right] \\
& =\left[\begin{array}{ccccccc}
0 & 0 & -s_{12} & \ldots & & -s_{1, n-1} & -s_{1 n} \\
0 & 2 s_{12} & s_{13} & \ldots & & s_{1 n}-s_{2, n-1} & -s_{2 n} \\
-s_{12} & s_{13} & 2 s_{23} & \ddots & & s_{2 n}-s_{3, n-1} & -s_{3 n} \\
\vdots & \vdots & \ddots & \ddots & \ddots & \vdots & \vdots \\
-s_{1, n-2} & s_{1, n-1}-s_{2, n-2} & & \ddots & 2 s_{n-2, n-1} & s_{n-2, n} & -s_{n-1, n} \\
-s_{1, n-1} & s_{1 n}-s_{2, n-1} & & \ldots & s_{n-2, n} & 2 s_{n-1, n} & 0 \\
-s_{1 n} & -s_{2 n} & & \ldots & -s_{n-1, n} & 0 & 0
\end{array}\right] .
\end{aligned}
$$

Similarly to (30), we reduce $B_{11}$ anti-diagonal-wise and the system of equations corresponding to each anti-diagonal is (31). Therefore we reduce $B_{11}$ to the form $0 x^{5}$.

The pair of blocks $\left(A_{21}, B_{21}\right)$ is reduced by adding $\delta\left(A_{21}, B_{21}\right)=\left(S_{22}^{T} F_{n}+\right.$ $\left.F_{n} S_{11}, S_{22}^{T} G_{n}+G_{n} S_{11}\right)$, where $S_{11}$ and $S_{22}$ are arbitrary matrices of the corresponding size. Obviously, that adding $S_{22}^{T} F_{n}+F_{n} S_{11}$ we reduce $A_{21}$ to zero. To preserve $A_{21}$, we must hereafter take $S_{11}$ and $S_{22}$ such that $F_{n} S_{11}=-S_{22}^{T} F_{n}$. Thus

$$
S_{11}=\left[\begin{array}{cccc} 
& & & 0 \\
& -S_{22}^{T} & & 0 \\
& & & \vdots \\
& & & 0 \\
-y_{1} & -y_{2} & \ldots & -y_{n+1}
\end{array}\right]
$$


and we reduce $B_{12}$ by adding

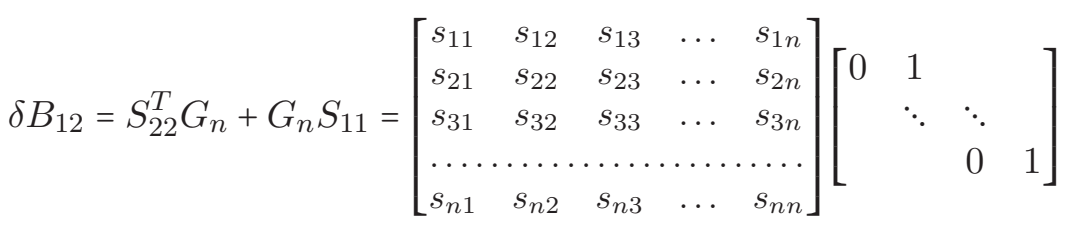

$$
\begin{aligned}
& +\left[\begin{array}{cccc}
0 & 1 & & \\
& \ddots & \ddots & \\
& & 0 & 1
\end{array}\right]\left[\begin{array}{cccccc}
-s_{11} & -s_{12} & -s_{13} & \ldots & -s_{1 n} & 0 \\
-s_{21} & -s_{22} & -s_{23} & \ldots & -s_{2 n} & 0 \\
-s_{31} & -s_{32} & -s_{33} & \ldots & -s_{3 n} & 0 \\
\ldots \ldots & \ldots \ldots & \ldots \ldots \ldots \ldots \ldots \ldots \ldots \ldots \\
-s_{n 1} & -s_{n 2} & -s_{n 3} & \ldots & -s_{n n} & 0 \\
y_{1} & y_{2} & y_{3} & \ldots & y_{n} & y_{n+1}
\end{array}\right]
\end{aligned}
$$

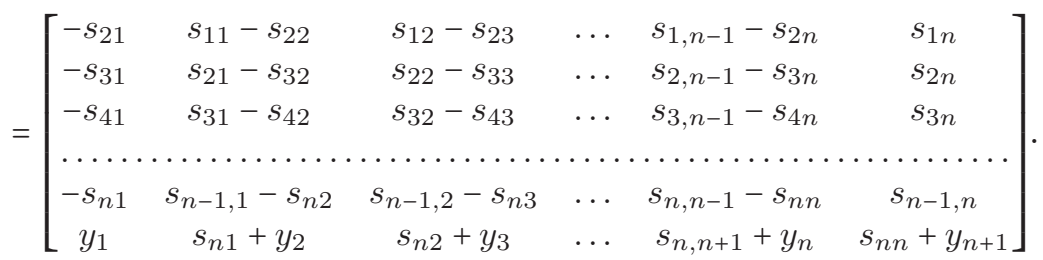

Clearly, we can set $B_{12}$ to zero by adding $\delta B_{12}$ (diagonal-wise).

The reduction of $\left(A_{21}, B_{21}\right)$ follows from the above, since $\left(A_{12}^{T}, B_{12}^{T}\right)=$ $\left(A_{21}, B_{21}\right)$ and $\left(\delta A_{12}^{T}, \delta B_{12}^{T}\right)=\left(\delta A_{21}, \delta B_{21}\right)$.

To the pair of blocks $\left(A_{22}, B_{22}\right)$ we can add $\delta\left(A_{22}, B_{22}\right)=\left(S_{12}^{T} F_{n}^{T}+\right.$ $\left.F_{n} S_{12}, S_{12}^{T} G_{n}^{T}+G_{n} S_{12}\right)$ in which $S_{12}$ is an arbitrary $(n+1)$-by-n matrix. Obviously, that adding $S_{21}^{T} F_{n}^{T}+F_{n} S_{21}$ we reduce each $n$-by- $n$ symmetric matrix $A_{22}$ to zero. To preserve $A_{22}$, we must hereafter take $S_{21}$ such that $F_{n} S_{12}=-S_{12}^{T} F_{n}^{T}$. Therefore

$$
S_{12}=\left[\begin{array}{ccccc}
0 & s_{12} & s_{13} & \ldots & s_{1 n} \\
-s_{12} & 0 & s_{23} & \ldots & s_{2 n} \\
-s_{13} & -s_{23} & 0 & \ldots & s_{3 n} \\
\vdots & \vdots & \vdots & \ddots & \vdots \\
-s_{1 n} & -s_{2 n} & -s_{3 n} & \ldots & 0 \\
s_{1, n+1} & s_{2, n+1} & s_{3, n+1} & \ldots & s_{n, n+1}
\end{array}\right]
$$

i.e. $S_{12}$ without the last row is skew symmetric. Now we reduce $B_{22}$ by 
adding

$$
\begin{aligned}
& \delta B_{22}=\left[\begin{array}{cccccc}
0 & -s_{12} & -s_{13} & \ldots & -s_{1 n} & s_{1, n+1} \\
s_{12} & 0 & -s_{23} & \ldots & -s_{2 n} & s_{2, n+1} \\
s_{13} & s_{23} & 0 & \ldots & -s_{3 n} & s_{3, n+1} \\
\vdots & \vdots & \vdots & \ddots & \vdots & \vdots \\
s_{1 n} & s_{2 n} & s_{3 n} & \ldots & 0 & s_{n, n+1}
\end{array}\right]\left[\begin{array}{ccc}
0 & & \\
1 & \ddots & \\
& \ddots & 0 \\
& & 1
\end{array}\right] \\
& +\left[\begin{array}{cccc}
0 & 1 & & \\
& \ddots & \ddots & \\
& & 0 & 1
\end{array}\right]\left[\begin{array}{ccccc}
0 & s_{12} & s_{13} & \ldots & s_{1 n} \\
-s_{12} & 0 & s_{23} & \ldots & s_{2 n} \\
-s_{13} & -s_{23} & 0 & \ldots & s_{3 n} \\
\vdots & \vdots & \vdots & \ddots & \vdots \\
-s_{1 n} & -s_{2 n} & -s_{3 n} & \ldots & 0 \\
s_{1, n+1} & s_{2, n+1} & s_{3, n+1} & \ldots & s_{n, n+1}
\end{array}\right] \\
& =\left[\begin{array}{ccccc}
-2 s_{12} & -s_{13} & \ldots & -s_{1 n}+s_{2, n-1} & s_{1, n+1}+s_{2 n} \\
-s_{13} & -2 s_{23} & \ldots & -s_{2 n}+s_{3, n-1} & s_{2, n+1}+s_{3 n} \\
\vdots & \vdots & \ddots & \vdots & \vdots \\
-s_{1 n}+s_{2, n-1} & -s_{2 n}+s_{3, n-1} & \ldots & -2 s_{n-1, n} & s_{n-1, n+1} \\
s_{1, n+1}+s_{2 n} & s_{2, n+1}+s_{3 n} & \ldots & s_{n-1, n+1} & 2 s_{n, n+1}
\end{array}\right] .
\end{aligned}
$$

Since each anti-diagonal of $\delta B_{22}$ has unique variables, we reduce $B_{22}$ antidiagonal-wise. For each half of any anti-diagonal we have the system of equations ((31) without the first equation), which has a solution. Therefore we reduce each anti-diagonal of $\left(A_{22}, B_{22}\right)$ to zero and so we reduce $\left(A_{22}, B_{22}\right)$ to zero.

Hence $\mathcal{D}\left(\mathcal{L}_{m}\right)$ is equal to $(15)$.

3.3. Off-diagonal blocks of matrices of $\mathcal{D}$ that correspond to summands of $(A, B)_{\text {can }}$ of the same type

In this section we check the condition (ii) of Lemma 3.2 for off-diagonal blocks of $\mathcal{D}$ defined in Theorem 2.1(ii). The horizontal and vertical strips of these diagonal blocks contain summands of $(A, B)_{\text {can }}$ of the same type.

\subsubsection{Pairs of blocks $\mathcal{D}\left(\mathcal{H}_{n}(\mu), \mathcal{H}_{m}(\lambda)\right)$ and $\mathcal{D}\left(\mathcal{K}_{n}, \mathcal{K}_{m}\right)$}

Due to Lemma 3.2(ii), it suffices to prove that each group of four matrices $\left((A, B),\left(A^{T}, B^{T}\right)\right)$ can be reduced to exactly one group of the form (16) (or, respectively (17)) by adding

$$
\left(R^{T} \mathcal{H}_{m}(\lambda)+\mathcal{H}_{n}(\mu) S, S^{T} \mathcal{H}_{n}(\mu)+\mathcal{H}_{m}(\lambda) R\right), \quad S \in \mathbb{C}^{n \times m}, R \in \mathbb{C}^{m \times n} .
$$

Obviously, if we reduce $(A, B)$ then $\left(A^{T}, B^{T}\right)$ is reduced automatically. The matrix pair $(A, B)$ is reduced by adding

$$
\delta(A, B)=R^{T} \mathcal{H}_{m}(\lambda)+\mathcal{H}_{n}(\mu) S=\left(R^{T} \Delta_{m}+\Delta_{n} S, R^{T} \Lambda_{m}(\lambda)+\Lambda_{n}(\mu) S\right) .
$$


It is clear that we can set $A$ to zero. To preserve $A$, we must hereafter take $R$ and $S$ such that

$$
R^{T} \Delta_{m}+\Delta_{n} S=0 \Leftrightarrow R^{T}=-\Delta_{n} S \Delta_{m} .
$$

It follows that $B$ is reduced by adding

$$
\begin{aligned}
\delta B=R^{T} \Lambda_{m}(\lambda)+\Lambda_{n}(\mu) S=-\Delta_{n} S \Delta_{m} \Lambda_{m}(\lambda)+\Lambda_{n}(\mu) S \\
= \begin{cases}(\lambda-\mu) s_{n-i+1, j}-s_{n-i+1, j-1}+s_{n-i+2, j} & \text { if } 2 \leq i \leq n, \quad 2 \leq j \leq m, \\
(\lambda-\mu) s_{n-i+1, j}-s_{n-i+1, j-1} & \text { if } 2 \leq j \leq m, \quad i=1, \\
(\lambda-\mu) s_{n-i+1, j}+s_{n-i+2, j} & \text { if } 2 \leq i \leq n, \quad j=1, \\
(\lambda-\mu) s_{n 1} & \text { if } i=j=1 .\end{cases}
\end{aligned}
$$

We have the system of $n m$ equations that has a solution if $\lambda \neq \mu$. Hence in the case $\lambda \neq \mu$ we can set any pair $(A, B)$ of $n$-by- $m$ matrices to zero.

For the case $\lambda=\mu$ we have

$$
\begin{aligned}
& \delta B=R^{T} \Lambda_{m}(\lambda)+\Lambda_{n}(\lambda) S=-\Delta_{n} S \Delta_{m} \Lambda_{m}(\lambda)+\Lambda_{n}(\lambda) S
\end{aligned}
$$

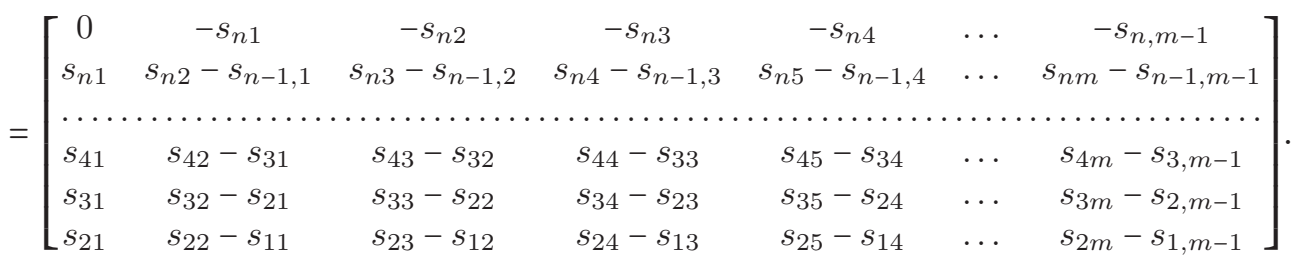

By adding (anti-diagonal-wise) $\delta B$ we reduce $B$ to the form $0^{\star}$.

We have shown that $\mathcal{D}\left(\mathcal{H}_{m}(\mu), \mathcal{H}_{n}(\lambda)\right)$ is equal to (16) as well as that $\mathcal{D}\left(\mathcal{K}_{m}, \mathcal{K}_{n}\right)$ is equal to (17).

\subsubsection{Pairs of blocks $\mathcal{D}\left(\mathcal{L}_{n}, \mathcal{L}_{m}\right)$}

Due to Lemma 3.2(ii), it suffices to show that each four matrices $\left((A, B),\left(A^{T}, B^{T}\right)\right)$ can be reduced to the form (18) by adding

$$
\left(R^{T} \mathcal{L}_{m}+\mathcal{L}_{n} S, S^{T} \mathcal{L}_{n}+\mathcal{L}_{m} R\right), \quad S \in \mathbb{C}^{2 n+1 \times 2 m+1}, R \in \mathbb{C}^{2 m+1 \times 2 n+1} .
$$

It is enough to reduce only $(A, B)$ and the pair $\left(A^{T}, B^{T}\right)$ is reduced automatically.

$$
\begin{aligned}
& \delta(A, B)=\left(\left[\begin{array}{ll}
\delta A_{11} & \delta A_{12} \\
\delta A_{21} & \delta A_{22}
\end{array}\right],\left[\begin{array}{ll}
\delta B_{11} & \delta B_{12} \\
\delta B_{21} & \delta B_{22}
\end{array}\right]\right)=R^{T} \mathcal{L}_{m}+\mathcal{L}_{n} S \\
& =\left(R^{T}\left[\begin{array}{cc}
0 & F_{m}^{T} \\
F_{m} & 0
\end{array}\right]+\left[\begin{array}{cc}
0 & F_{n}^{T} \\
F_{n} & 0
\end{array}\right] S, R^{T}\left[\begin{array}{cc}
0 & G_{m}^{T} \\
G_{m} & 0
\end{array}\right]+\left[\begin{array}{cc}
0 & G_{n}^{T} \\
G_{n} & 0
\end{array}\right] S\right) \\
& =\left(\left[\begin{array}{cc}
R_{12}^{T} F_{m}+F_{n}^{T} S_{21} & R_{11}^{T} F_{m}^{T}+F_{n}^{T} S_{22} \\
R_{22}^{T} F_{m}+F_{n} S_{11} & R_{21}^{T} F_{m}^{T}+F_{n} S_{12}
\end{array}\right],\left[\begin{array}{ll}
R_{12}^{T} G_{m}+G_{n}^{T} S_{21} & R_{11}^{T} G_{m}^{T}+G_{n}^{T} S_{22} \\
R_{22}^{T} G_{m}+G_{n} S_{11} & R_{21}^{T} G_{m}^{T}+G_{n} S_{12}
\end{array}\right]\right) .
\end{aligned}
$$


First we reduce the pair $\left(A_{11}, B_{11}\right)$. Easy to see that by adding $\delta A_{11}$ we can reduce $A_{11}$ to $0_{*}$. To preserve $A_{11}$, we must hereafter take $R_{12}$ and $S_{21}$ such that $R_{12}^{T} F_{m}=-F_{n}^{T} S_{21}$. This means that

$$
R_{12}^{T}=\left[\begin{array}{ccc} 
& -Q & \\
0 & \ldots & 0
\end{array}\right] \text { and } S_{21}=\left[\begin{array}{cc}
0 \\
Q & \vdots \\
& 0
\end{array}\right] \text {, where } Q \text { is any } n \text {-by- } m \text { matrix. }
$$

Hence

$$
\begin{aligned}
& \delta B_{11}=R_{12}^{T} G_{m}+G_{n}^{T} S_{21}=\left[\begin{array}{ccc} 
& -Q & \\
0 & \ldots & 0
\end{array}\right] G_{m}+G_{n}^{T}\left[\begin{array}{cc} 
& 0 \\
Q & \vdots \\
& 0
\end{array}\right]=
\end{aligned}
$$

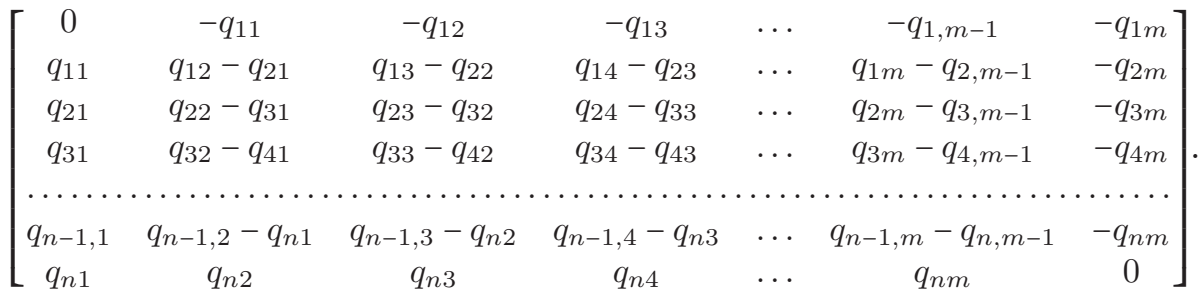

By adding $\delta B_{11}$ we can set each element of $B_{11}$ to zero except either the first column and the last row or the first row and the last column.

Now we consider $\left(A_{12}, B_{12}\right)$. We can set $A_{12}$ to zero by adding $\delta A_{12}$. To preserve $A_{12}$, we must hereafter take $R_{11}$ and $S_{22}$ such that $R_{11}^{T} F_{m}^{T}=-F_{n}^{T} S_{22}$. Thus

$$
R_{11}^{T}=\left[\begin{array}{cccc} 
& & & h_{1} \\
& -S_{22} & & \vdots \\
0 & \cdots & 0 & h_{n+1}
\end{array}\right],
$$

where $S_{22}$ is any $n$-by- $m$ matrix. Therefore

$$
\begin{aligned}
& \delta B_{12}=R_{11}^{T} G_{m}^{T}+G_{n}^{T} S_{22}=\left[\begin{array}{cccc} 
& & h_{1} \\
& -S_{22} & & \vdots \\
0 & \ldots & 0 & h_{n+1}
\end{array}\right] G_{m}^{T}+G_{n}^{T} S_{22}=
\end{aligned}
$$

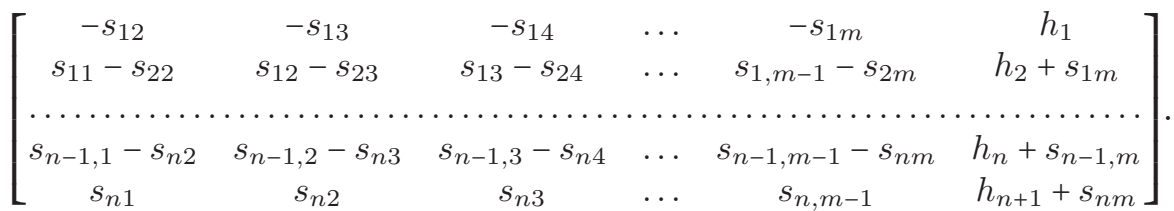

If $n \geq m-1$ then we can set $B_{12}$ to zero by adding $\delta B_{12}$. If $n<m-1$ then we cannot set the block $B_{12}$ to zero. Then we start the diagonal-wise reduction from the down left hand corner and set the first $(n-1)$ diagonals of $B_{12}$ to zero. We set the next $(m-n)$ diagonals of $B_{12}$ to zero, except the last 
element of each of them. The remaining $n$ diagonals we can set to zero too. Hence we reduce this pair of blocks $\left(A_{12}, B_{12}\right)$ to the form $\left(0,0_{n+1, m}^{\boxminus}\right)$.

Now we reduce $\left(A_{21}, B_{21}\right)$. We can set $A_{21}$ to zero by adding $\delta A_{21}$. To preserve $A_{21}$, we must hereafter take $R_{22}$ and $S_{11}$ such that $R_{22}^{T} F_{m}=-F_{n} S_{11}$, i.e.

$$
S_{11}=\left[\begin{array}{ccc} 
& & 0 \\
& -R_{22}^{T} & \vdots \\
h_{1} & \ldots & h_{m+1}
\end{array}\right],
$$

where $R_{22}^{T}$ is any $n$-by- $m$ matrix. Therefore

$$
\begin{aligned}
& \delta B_{21}=R_{22}^{T} G_{m}+G_{n} S_{11}=R_{22}^{T} G_{m}+G_{n}\left[\begin{array}{ccc} 
& & 0 \\
& -R_{22}^{T} & \vdots \\
& & 0 \\
h_{1} & \ldots & h_{m+1}
\end{array}\right]
\end{aligned}
$$

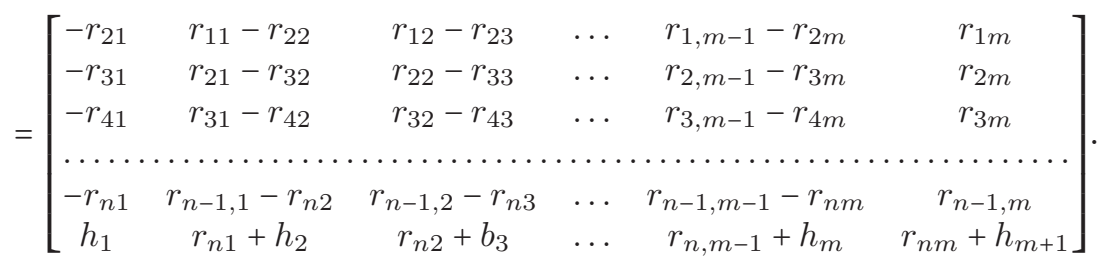

If $m+1 \geq n$ then we can set $B_{21}$ to zero by adding $\delta B_{21}$. If $m+1<n$ then we cannot set the whole $B_{21}$ to zero. By adding $\delta\left(A_{21}, B_{21}\right)$ and arguing as in the previous case we reduce $\left(A_{21}, B_{21}\right)$ to $\left(0,0_{m+1, n}^{\boxminus T}\right)$.

Now let us consider the pair $\left(A_{22}, B_{22}\right)$. We can set $A_{22}$ to zero by adding $\delta A_{22}$. To preserve $A_{22}$, we must hereafter take $R_{21}$ and $S_{12}$ such that $R_{21}^{T} F_{m}^{T}=$ $-F_{n} S_{12}$, i.e.

$$
S_{12}=\left[\begin{array}{ccc} 
& -Q & \\
g_{1} & \cdots & g_{m}
\end{array}\right] \text { and } R_{21}^{T}=\left[\begin{array}{cc} 
& h_{1} \\
Q & \vdots \\
& h_{n}
\end{array}\right] \text {, where } Q \text { is any } n \text {-by- } m \text { matrix. }
$$

It follows that

$$
\begin{aligned}
& \delta B_{22}=R_{21}^{T} G_{m}^{T}+G_{n} S_{12}=\left[\begin{array}{cc} 
& h_{1} \\
Q & \vdots \\
& h_{n}
\end{array}\right] G_{m}^{T}+G_{n}\left[\begin{array}{ccc} 
& -Q & \\
g_{1} & \cdots & g_{m}
\end{array}\right]
\end{aligned}
$$

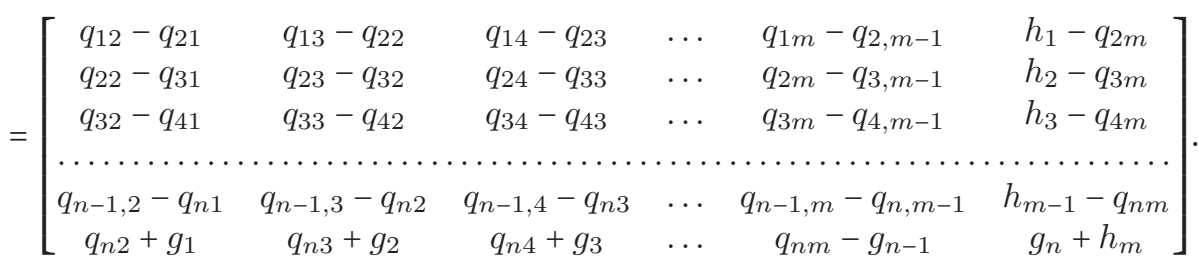


We can set each anti-diagonal of $B_{22}$ to zero independently. Thus adding $\delta B_{22}$ we reduce $B_{22}$ to zero.

Hence $\mathcal{D}\left(\mathcal{L}_{m}, \mathcal{L}_{n}\right)$ has the form (18).

3.4. Off-diagonal blocks of matrices of $\mathcal{D}$ that correspond to summands of $(A, B)_{\text {can }}$ of different types

At last, we check the condition (ii) of Lemma 3.2 for off-diagonal blocks of $\mathcal{D}$ defined in Theorem 2.1(iii); the diagonal blocks of their horizontal and vertical strips contain summands of $(A, B)_{\text {can }}$ of different types.

\subsubsection{Pairs of blocks $\mathcal{D}\left(\mathcal{H}_{n}(\lambda), \mathcal{K}_{m}\right)$}

Due to Lemma 3.2(ii), it suffices to prove that each group of four matrices $\left((A, B),\left(A^{T}, B^{T}\right)\right)$ can be reduced to exactly one group of the form (19) by adding

$$
\left(R^{T} \mathcal{K}_{m}+\mathcal{H}_{n}(\lambda) S, S^{T} \mathcal{H}_{n}(\lambda)+\mathcal{K}_{m} R\right), \quad S \in \mathbb{C}^{n \times m}, R \in \mathbb{C}^{m \times n} .
$$

Clearly, we can reduce only $(A, B)$ and the pair $\left(A^{T}, B^{T}\right)$ is reduced automatically. We have

$\delta(A, B)=(\delta A, \delta B)=R^{T} \mathcal{K}_{m}+\mathcal{H}_{n}(\lambda) S=\left(R^{T} \Lambda_{m}(0)+\Delta_{n} S, R^{T} \Delta_{m}+\Lambda_{n}(\lambda) S\right)$.

We can set $A$ to zero by adding $\delta A$. To preserve $A$, we must hereafter take $R$ and $S$ such that

$$
R^{T} \Lambda_{m}(0)+\Delta_{n} S=0 \Rightarrow S=-\Delta_{n} R^{T} \Lambda_{m}(0) .
$$

Thus $B$ is reduced to zero by adding

$$
\delta B=R^{T} \Delta_{m}+\Lambda_{n}(\lambda) S=R^{T} \Delta_{m}-\Lambda_{n}(\lambda) \Delta_{n} R^{T} \Lambda_{m}(0) .
$$

Hence $\mathcal{D}\left(\mathcal{H}_{n}(\lambda), \mathcal{K}_{m}\right)$ is equal to zero.

\subsubsection{Pairs of blocks $\mathcal{D}\left(\mathcal{H}_{n}(\lambda), \mathcal{L}_{m}\right)$}

Due to Lemma 3.2(ii), it suffices to prove that each group of four matrices $\left((A, B),\left(A^{T}, B^{T}\right)\right)$ can be reduced to (20) by adding

$$
\left(R^{T} \mathcal{L}_{m}+\mathcal{H}_{n}(\lambda) S, S^{T} \mathcal{H}_{n}(\lambda)+\mathcal{L}_{m} R\right), \quad S \in \mathbb{C}^{n \times 2 m+1}, R \in \mathbb{C}^{2 m+1 \times n} .
$$

Obviously, that we can reduce only $(A, B)$ and the pair $\left(A^{T}, B^{T}\right)$ is reduced automatically. We have

$$
\delta(A, B)=R^{T} \mathcal{L}_{m}+\mathcal{H}_{n}(\lambda) S=\left(R^{T}\left[\begin{array}{cc}
0 & F_{m}^{T} \\
F_{m} & 0
\end{array}\right]+\Delta_{n} S, R^{T}\left[\begin{array}{cc}
0 & G_{m}^{T} \\
G_{m} & 0
\end{array}\right]+\Lambda_{n}(\lambda) S\right) .
$$


It is clear that we can set $A$ to zero. To preserve $A$, we must hereafter take $R$ and $S$ such that

$$
R^{T}\left[\begin{array}{cc}
0 & F_{m}^{T} \\
F_{m} & 0
\end{array}\right]+\Delta_{n} S=0 \Rightarrow S=-\Delta_{n}\left[\begin{array}{cc}
R_{11}^{T} & R_{21}^{T} \\
R_{12}^{T} & R_{22}^{T}
\end{array}\right]\left[\begin{array}{cc}
0 & F_{m}^{T} \\
F_{m} & 0
\end{array}\right] .
$$

Hence $B$ is reduced by adding

$$
\begin{aligned}
\delta B= & R^{T}\left[\begin{array}{cc}
0 & G_{m}^{T} \\
G_{m} & 0
\end{array}\right]-\Lambda_{n}(\lambda) \Delta_{n} R^{T}\left[\begin{array}{cc}
0 & F_{m}^{T} \\
F_{m} & 0
\end{array}\right] \\
& =\left\{\begin{array}{lll}
-\lambda r_{i, n-1}-r_{i-1, n-1} & \text { if } 1 \leq j \leq n, \quad j=1, \\
-\lambda r_{i, m+1+j}-r_{i-1, m+1+j}+r_{i, m+j} & \text { if } 1 \leq i \leq n, \quad 1<j<m+1, \\
r_{i n} & \text { if } 1 \leq i \leq n, \quad j=m+1, \\
-\lambda r_{i, j-m-1}-r_{i-1, j-m-1}+r_{i, j-m} & \text { if } 1 \leq i \leq n, \quad m+1<j \leq 2 m+1,
\end{array}\right.
\end{aligned}
$$

where we put $r_{0 t}:=0$. Adding $\delta B$ we reduce $B$ to the form $0 \leftarrow$.

Therefore $\mathcal{D}\left(\mathcal{H}_{n}(\lambda), \mathcal{L}_{m}\right)$ is equal to (20) .

\subsubsection{Pairs of blocks $\mathcal{D}\left(\mathcal{K}_{n}, \mathcal{L}_{m}\right)$}

Due to Lemma 3.2 (ii), it suffices to prove that each group of four matrices $\left((A, B),\left(A^{T}, B^{T}\right)\right)$ can be reduced to (21) by adding

$$
\left(R^{T} \mathcal{L}_{m}+\mathcal{K}_{n} S, S^{T} \mathcal{K}_{n}+\mathcal{L}_{m} R\right), \quad S \in \mathbb{C}^{n \times 2 m+1}, R \in \mathbb{C}^{2 m+1 \times n} .
$$

As before, we can reduce only $(A, B)$ and the pair $\left(A^{T}, B^{T}\right)$ is reduced automatically. We have

$$
\delta(A, B)=R^{T} \mathcal{L}_{m}+\mathcal{K}_{n} S=\left(R^{T}\left[\begin{array}{cc}
0 & F_{m}^{T} \\
F_{m} & 0
\end{array}\right]+\Lambda_{n}(0) S, R^{T}\left[\begin{array}{cc}
0 & G_{m}^{T} \\
G_{m} & 0
\end{array}\right]+\Delta_{n} S\right) .
$$

It is easy to check that we can set $B$ to zero. To preserve $B$, we must hereafter take $R$ and $S$ such that

$$
R^{T}\left[\begin{array}{cc}
0 & G_{m}^{T} \\
G_{m} & 0
\end{array}\right]+\Delta_{n} S=0 \Rightarrow S=-\Delta_{n}\left[\begin{array}{cc}
R_{11}^{T} & R_{21}^{T} \\
R_{12}^{T} & R_{22}^{T}
\end{array}\right]\left[\begin{array}{cc}
0 & G_{m}^{T} \\
G_{m} & 0
\end{array}\right] .
$$

Thus $A$ is reduced by adding

$$
\begin{aligned}
\delta A=R^{T}\left[\begin{array}{cc}
0 & F_{m}^{T} \\
F_{m} & 0
\end{array}\right]-\Lambda_{n}(0) \Delta_{n} R^{T}\left[\begin{array}{cc}
0 & G_{m}^{T} \\
G_{m} & 0
\end{array}\right] \\
=\left\{\begin{array}{lll}
r_{i n-1} & \text { if } 1 \leq j \leq n, \quad j=1, \\
r_{i, m+1+j}-r_{i-1, m+j} & \text { if } 1 \leq i \leq n, \quad 1<j<m+1, \\
r_{i-1, n} & \text { if } 1 \leq i \leq n, \quad j=m+1, \\
r_{i, j-m-1}-r_{i-1, j-m} & \text { if } 1 \leq i \leq n, \quad m+1<j \leq 2 m+1,
\end{array}\right.
\end{aligned}
$$

where we put $r_{0 t}:=0$.

Therefore $\mathcal{D}\left(\mathcal{K}_{n}, \mathcal{L}_{m}\right)$ is equal to (21). 


\section{Acknowledgements}

The author is thankful to Vladimir V. Sergeichuk for introducing him to the area of miniversal deformations and, in particular, the problem considered in this paper. The author also thanks to the anonymous referee for the helpful suggestions.

The work was supported by the Swedish Research Council (VR) under grant E0485301, and by eSSENCE, a strategic collaborative e-Science programme funded by the Swedish Research Council.

\section{References}

[1] E. Antoniou and S. Vologiannidis. A new family of companion forms of polynomial matrices. Electron. J. Linear Algebra, 11:78-87, 2004.

[2] V. Arnold. On matrices depending on parameters. Russian Math. Surveys, 26:29-43, 1971.

[3] V. Arnold. Geometrical methods in the theory of ordinary differential equations. Springer-Verlag, New York, 2nd edition, 1997.

[4] T. Berger, G. Halikias, and N. Karcanias. Effects of dynamic and non-dynamic element changes in RC and RL networks. Int. J. Circ. Theor. Appl., 43(1):36-59, 2015.

[5] V. A. Bovdi, M. A. Salim, and V. V. Sergeichuk. Neighborhood radius estimation for Arnold's miniversal deformations of complex and p-adic matrices. Linear Algebra Appl., 512:97-112, 2017.

[6] T. Brüll and V. Mehrmann. STCSSP: A FORTRAN 77 routine to compute a structured staircase form for a (skew-)symmetric/(skew-)symmetric matrix pencil. Preprint 31-2007, Institut für Mathematik, TU Berlin, 2007.

[7] A. Dmytryshyn. Miniversal deformations of pairs of skew-symmetric matrices under congruence. Linear Algebra Appl., 506:506-534, 2016.

[8] A. Dmytryshyn. Structure preserving stratification of skew-symmetric matrix polynomials. Linear Algebra Appl., 532:266-286, 2017.

[9] A. Dmytryshyn, V. Futorny, B. Kågström, L. Klimenko, and V. Sergeichuk. Change of the congruence canonical form of 2-by-2 and 3-by-3 matrices under perturbations and bundles of matrices under congruence. Linear Algebra Appl., 469:305-334, 2015.

[10] A. Dmytryshyn, V. Futorny, and V. Sergeichuk. Miniversal deformations of matrices of bilinear forms. Linear Algebra Appl., 436:2670-2700, 2012.

[11] A. Dmytryshyn, V. Futorny, and V. Sergeichuk. Miniversal deformations of matrices under * congruence and reducing transformations. Linear Algebra Appl., 446:388-420, 2014. 
[12] A. Dmytryshyn, S. Johansson, and B. Kågström. Codimension computations of congruence orbits of matrices, symmetric and skew-symmetric matrix pencils using Matlab. Technical Report UMINF 13.18, Department of Computing Science, Umeå University, Sweden, 2013.

[13] A. Dmytryshyn, S. Johansson, and B. Kågström. Canonical structure transitions of system pencils. SIAM J. Matrix Anal. Appl., 38(4):1249-1267, 2017.

[14] A. Dmytryshyn, S. Johansson, B. Kågström, and P. Van Dooren. Geometry of spaces for matrix polynomial Fiedler linearizations. UMINF 15.17, Dept. of Computing Science, Umeå University, Sweden, 2015.

[15] A. Dmytryshyn and B. Kågström. Orbit closure hierarchies of skew-symmetric matrix pencils. SIAM J. Matrix Anal. Appl., 35(4):1429-1443, 2014.

[16] A. Dmytryshyn, B. Kågström, and V. Sergeichuk. Symmetric matrix pencils: codimension counts and the solution of a pair of matrix equations. Electron. J. Linear Algebra, 27:1-18, 2014.

[17] N. A. Dumont. On the solution of generalized non-linear complex-symmetric eigenvalue problems. Internat. J. Numer. Methods Engrg., 71(13):1534-1568, 2007.

[18] A. Edelman, E. Elmroth, and B. Kågström. A geometric approach to perturbation theory of matrices and matrix pencils. Part I: Versal deformations. SIAM J. Matrix Anal. Appl., 18(3):653-692, 1997.

[19] A. Edelman, E. Elmroth, and B. Kågström. A geometric approach to perturbation theory of matrices and matrix pencils. Part II: A stratification-enhanced staircase algorithm. SIAM J. Matrix Anal. Appl., 20(3):667-669, 1999.

[20] V. Futorny, V. Klimenko, and V. Sergeichuk. Change of the * congruence canonical form of 2-by-2 matrices under perturbations. Electron. J. Linear Algebra, 27, 2014.

[21] D. M. Galin. Real matrices depending on parameters. Uspehi Mat. Nauk, 27(1(163)):241-242, 1972.

[22] M. García-Planas and V. Sergeichuk. Simplest miniversal deformations of matrices, matrix pencils, and contragredient matrix pencils. Linear Algebra Appl., 302-303:4561, 1999 .

[23] N. Higham, D. S. Mackey, M. N., and F. Tisseur. Symmetric linearizations for matrix polynomials. SIAM J. Matrix Anal. Appl., 29(1):143-159, 2007.

[24] P. Johansson. Matrix Canonical Structure Toolbox. Technical Report UMINF 06.15, Department of Computing Science, Umeå University, Sweden, 2006.

[25] S. Johansson, B. Kågström, and P. Van Dooren. Stratification of full rank polynomial matrices. Linear Algebra Appl., 439:1062-1090, 2013.

[26] B. Kågström, S. Johansson, and P. Johansson. StratiGraph Tool: Matrix Stratification in Control Applications. In L. Biegler, S. Campbell, and V. Mehrmann, editors, Control and Optimization with Differential-Algebraic Constraints, chapter 5. SIAM Publications, 2012. 
[27] A. Mailybaev and A. Seyranian. On the boundaries of the parametric resonance domain. J. Appl. Math. Mech., 64(6):909 - 923, 2000.

[28] A. A. Mailybaev. Transformation of families of matrices to normal forms and its application to stability theory. SIAM J. Matrix Anal. Appl., 21(2):396-417, 2000.

[29] A. A. Mailybaev and A. P. Seyranian. On singularities of a boundary of the stability domain. SIAM J. Matrix Anal. Appl., 21(1):106-128, 1999.

[30] C. Mehl, V. Mehrmann, and M. Wojtylak. Parameter-dependent rank-one perturbations of singular Hermitian or symmetric pencils. SIAM J. Matrix Anal. Appl., 38(1):72-95, 2017.

[31] B. N. Parlett. Symmetric matrix pencils. J. Comput. Appl. Math., 38(1):373 - 385, 1991.

[32] R. C. Thompson. Pencils of complex and real symmetric and skew matrices. Linear Algebra Appl., 147:323-371, 1991.

[33] F. Tisseur and K. Meerbergen. The quadratic eigenvalue problem. SIAM Review, 43(2):235-286, 2001. 